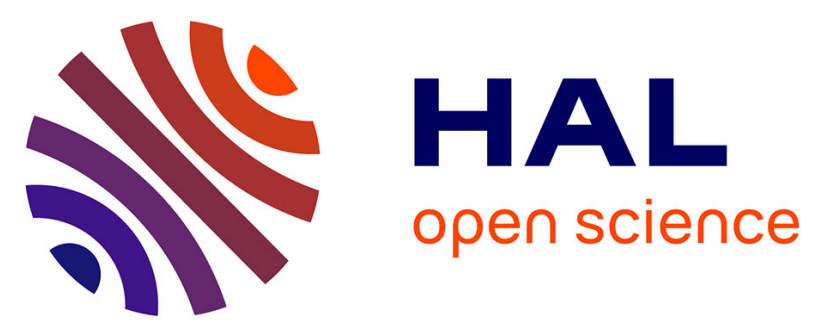

\title{
Intra-operative Registration for Stereotactic Procedures driven by a combined Biomechanical Brain and CSF Model
}

\author{
Alexandre Bilger, Éric Bardinet, Sara Fernández-Vidal, Christian Duriez, \\ Pierre Jannin, Stéphane Cotin
}

\section{To cite this version:}

Alexandre Bilger, Éric Bardinet, Sara Fernández-Vidal, Christian Duriez, Pierre Jannin, et al.. Intraoperative Registration for Stereotactic Procedures driven by a combined Biomechanical Brain and CSF Model. ISBMS - International Symposium on Biomedical Simulation, Oct 2014, Strasbourg, France. pp.76-85, 10.1007/978-3-319-12057-7_9. hal-01058435

\section{HAL Id: hal-01058435 \\ https://hal.inria.fr/hal-01058435}

Submitted on 26 Aug 2014

HAL is a multi-disciplinary open access archive for the deposit and dissemination of scientific research documents, whether they are published or not. The documents may come from teaching and research institutions in France or abroad, or from public or private research centers.
L'archive ouverte pluridisciplinaire HAL, est destinée au dépôt et à la diffusion de documents scientifiques de niveau recherche, publiés ou non, émanant des établissements d'enseignement et de recherche français ou étrangers, des laboratoires publics ou privés. 


\title{
Intra-operative Registration for Stereotactic Procedures driven by a combined Biomechanical Brain and CSF Model
}

\author{
Alexandre Bilger ${ }^{1}$, Éric Bardinet ${ }^{2}$, Sara Fernandez-Vidal ${ }^{2}$, Christian Duriez $^{1}$, \\ Pierre Jannin ${ }^{3}$, and Stéphane Cotin ${ }^{1}$ \\ 1 Inria Lille - Nord Europe Research Centre \\ 2 Centre de Recherche de l'Institut du Cerveau et de la Moelle épiniere, UMR-S975, \\ Paris; Inserm \\ 3 Equipe Medicis, U1099 LTSI, Université Rennes I
}

\begin{abstract}
During stereotactic neurosurgery, the brain shift could affect the accuracy of the procedure. However, this deformation of the brain is not often considered in the pre-operative planning step or intraoperatively, and may lead to surgical complications, side effects or ineffectiveness. In this paper, we present a method to update the preoperative planning based on a physical simulation of the brain shift. Because the simulation requires unknown input parameters, the method relies on a parameter estimation process to compute the intracranial state that matches the partial data taken from intra-operative modalities. The simulation is based on a biomechanical model of the brain and the cerebro-spinal fluid. In this paper, we show on an anatomical atlas that the method is numerically sound.
\end{abstract}

\section{Introduction}

Stereotactic neurosurgery requires a high precision for target location and target definition. For example, one of the main targets in deep brain stimulation, the subthalamic nucleus, is about 9 millimeters long. However, a combination of deformation and motion of the brain, known as brain shift, can occur during the procedure, depending on the surgical technique and the patient's brain anatomy and pathology. In stereotactic procedures, it may cause a displacement of the target or other structures. Elias et al.4] reported an anterior commissure shift up to $5.67 \mathrm{~mm}$ with a mean of $0.98 \mathrm{~mm}$, and higher shift values for the frontal pole.

Because brain shift is a major source of errors when applying the planned strategy intra-operatively, several groups have studied the problem of brain shift compensation using a deformable model, instead of using a fully intensity-based method. Skrinjar et al. 8 presented a method to deform pre-operative data according to a partial intra-operative brain surface, captured by a stereo camera, while Chen et al. [3] and Audette et al.[1] used a laser range scanner. However the equipment is not standard and requires a large working space without occlusion, which is not always the case in neurosurgery. The work of Wittek et al. [10] 


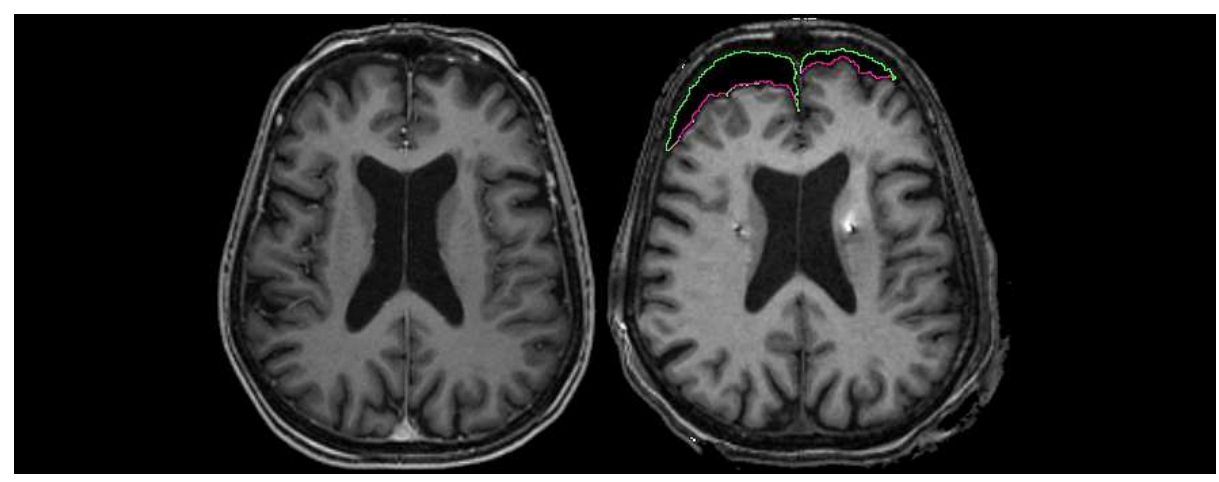

Fig. 1: Pre-operative (left) MR image without brain shift, and post-operative (right) MRI showing asymmetric brain shift. The green and pink contours is a segmentation of the pneumocephalus (air inside skull). The pink line represents the surface of the brain in contact with air.

is very similar to 8 but they used intra-operative MRI to guide the deformable model and took extra care on the complex model of the brain tissue deformation. However [8] and [10] do not model physically the brain shift phenomenon, because they include artificial forces by adding virtual springs or constraints between pre- and intra-operative control points. None of these methods accounts for the effects of gravity, interaction of CSF and brain tissue and loss of CSF. On the contrary, Chen et al. 3 used a computational model which accounts for CSF loss and gravity. They pre-operatively built a statistical atlas of deformation to solve the inverse problem intra-operatively.

We propose a new method to take into account brain shift during a stereotactic neurosurgical procedure. It relies on high resolution pre-operative MR image and intra-operative image (CT scan) of the patient's brain, acquired once the brain has shifted, but the method could be extended to other modalities such as intra-operative MRI. The pre-operative data are deformed according to a physical model of brain shift, mainly based on a cerebro-spinal fluid (CSF) interaction with the brain tissue, gravity and brain-skull interactions. To best fit the intra-operative configuration, the input parameters of the brain shift model are estimated intra-operatively with a measure of similarity with a series of precomputed deformed configurations. The output of this inverse problem gives parameters used to compute the intra-operative configuration, as detailed as the pre-operative data. With this information, the surgeon is able to update the pre-operative planning data (target coordinates, trajectory angles etc).

The following section describes the brain shift simulation and how we use it to solve the intra-operative registration based on a physical parameters estimation. Section 3 presents the results and the numerical validation on an anatomical atlas. Section 4 concludes and addresses future steps for the method. 


\section{Materials \& Methods}

The overall pipeline of our method is detailed in the figure 2 This section presents the model of brain shift used in the simulations and the parameters estimation (the highlighted steps in the figure 2). The other aspects are not treated in this paper as we focus on the numerical validation.

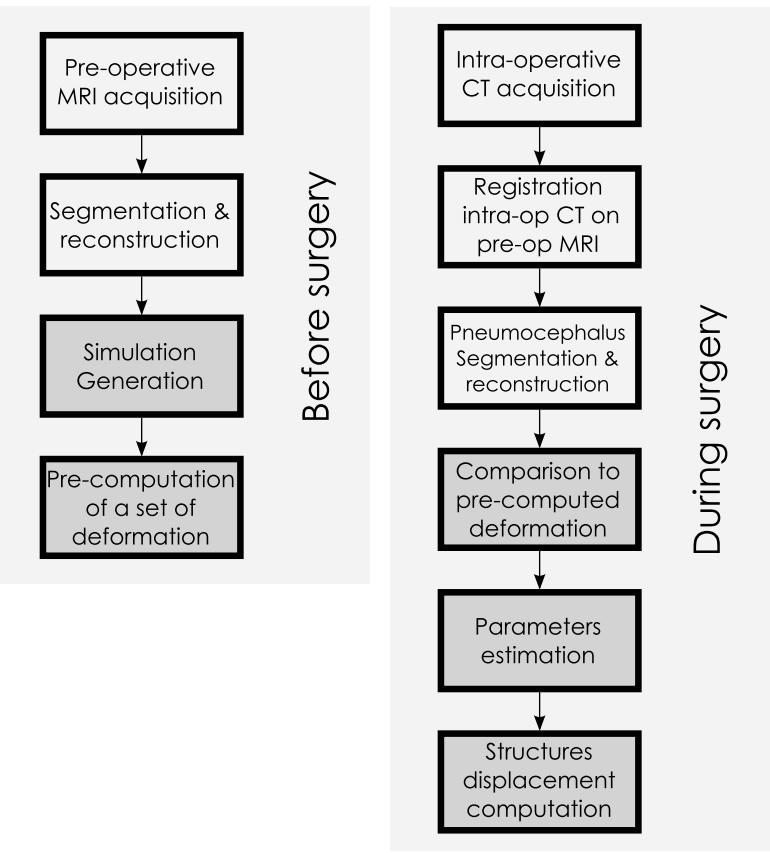

Fig. 2: Steps of our method (read from left to right, then from top to bottom): the acquired pre-operative MRI enables to generate a simulation. A set of deformation is pre-computed. During the surgery, the pneumocephalus is extracted from the intra-operative CT, rigidly registered to the pre-operative data to be in the same space and compared to the set of pre-computed deformation. Finally, the displacement of the structures of interest is deduced. Among these steps, the most time consuming processes are done before the surgery.

\subsection{Presentation of the model}

The brain shift simulation relies on a physics-based model of the brain tissue deformation, the contact response with the skull and the falx cerebri, and the interaction with the CSF. It is based on the work of [2].

The brain deformation is computed using a non-linear geometric finite element method, with a linear constitutive law [5]. This allows for rotation in the 
model, while relying on a linear expression of the stress-strain relationship. The viscous part of the brain behavior is omitted, because we focus on the static equilibrium after the brain has shifted. The equation relating the external forces $f$ to the nodal displacements $u$ can be written as $f=K(u) u$, with $K$ the stiffness matrix depending on $u$. Both hemispheres are meshed independently with linear tetrahedrons.

When the brain deforms, the simulation algorithm solves the contacts with the skull and the falx cerebri, considered as rigid. Unilateral interpenetration constraints are created when a collision is detected. The area near the brainsterm is assigned fixed Dirichlet boundary conditions to impose a null displacement constraint. The contacts are solved with Lagrangian multipliers. Figure 3 shows the boundary conditions of the model.

The main cause of brain shift is a CSF loss [9. The resulting force of the surrounding fluid acting on the brain tissue acts against the weight, so that the brain is balanced. A CSF loss causes a fluid forces decrease, breaking the balance and leading to a brain shift. In order to cause a brain shift in our simulation, we include a model of CSF forces:

$$
\mathbf{f}_{C S F}=\iint_{S} \rho g h(P) \mathrm{d} \mathbf{S}
$$

where $\rho$ is the density of CSF (1000 $\left.\mathrm{kg} / \mathrm{m}^{3}\right), g$ is the norm of the gravity and $h$ is the distance between a point $P$ on the mesh and the fluid surface. The force $\mathbf{f}_{C S F}$ is applied onto every immersed triangle $S$ of the brain surface mesh. Asymmetric brain shift is observed on post-operative images (see Fig. 1). To handle this property, two independent models of fluid forces are present in the simulation, each acting independently on each hemispheres. With this model, the deformation depends also on the patient's head orientation compared to gravity direction, mechanical properties of the tissue and patient variability.

To recover the true rest configuration of the brain before applying gravity and external forces on the finite element model, we solve an inverse problem using an iterative geometric algorithm 7 . The algorithm is used with a static solver. The iterations are smoothed to handle the geometrical non-linearities of the deformation model.

\subsection{Non-rigid registration based on physical parameters estimation}

Parameters to estimate As explained in section 2.1, the brain shift model depends on the following parameters: patient's head orientation compared to gravity direction, patient variability (geometry), mechanical properties of the tissue and the amount of CSF lost during the procedure. The two first parameters are known: we can measure the orientation of the patient's head and the geometry is acquired by the segmentation of patient images. The mechanical parameters of the brain tissue have been estimated by several groups using different techniques, but there is no consensus and there is no direct measurement technique personalized for a patient. Although it would be interesting to add 


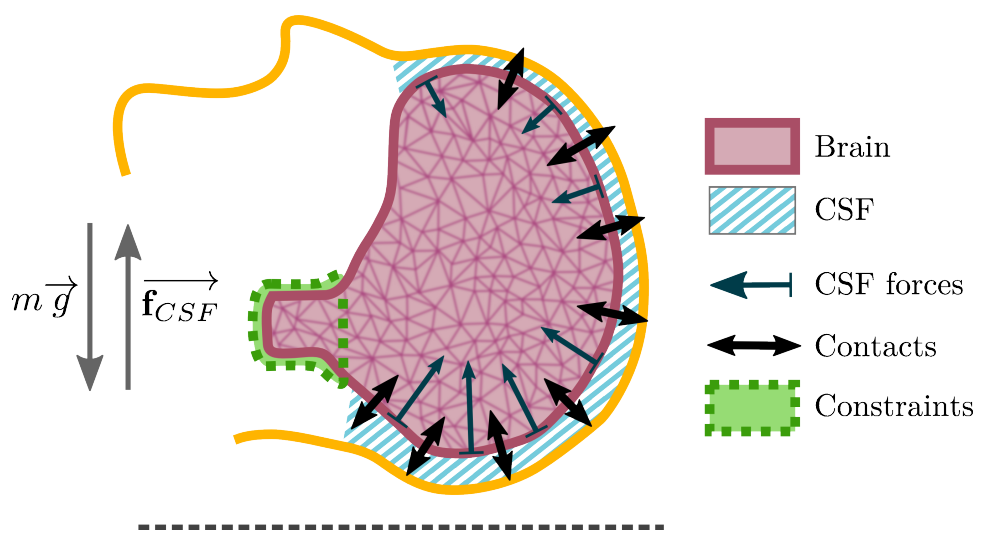

Fig. 3: Schematic representation of the brain model and the set of constraints describing its interactions with the environment. In this pre-operative configuration, the patient is in the supine position (see the gravity vector direction $\vec{g}$ ). The cerebro-spinal fluid surrounds the brain tissue and acts on it with pressure forces. The resultant force balances the weight (see the $\overrightarrow{\mathbf{f}_{C S F}}$ compared to the weight force $m \vec{g}$ ). The brain is under a null displacement constraint near the brainstem area. The illustration also shows the contacts between the brain and the skull.

the mechanical properties to the parameters to estimate, the deformation is also controlled by the CSF volume lost, meaning that these two parameters cannot be dissociated. That is why the mechanical properties are fixed to the value used in 6. Moreover, the mechanical parameters are not the physical cause of the brain shift phenomenon. We will show that an acceptable error in the mechanical parameters does affect the parameter estimation, but not the output of our algorithm. Finally, only the CSF volume lost is estimated. As we model both hemispheres independently, two quantities have to be estimated.

Error minimization In our algorithm, the only unknown input parameters are two volumes of CSF lost during the surgery. To estimate these parameters, we introduce a measure of similarity, to compare the final deformed geometry of the brain, obtained at equilibrium after applying a CSF loss in a simulation, and the visible surface of the brain, extracted from an intra-operative modality. The similarity between these two objects is defined with a least-squares approach:

$$
d(S, M)=\sum_{x_{S} \in S}\left\|x_{S}-p\left(x_{S}, M\right)\right\|^{2}
$$

with $x_{S}$ a point on the surface $S$ of the intra-operative brain, $p\left(x_{S}, M\right)$ is the projection of the point $x_{S}$ on the surface $M$ of the simulated brain. The measure is normalized by dividing by the number of points in $S$, and represents the 
average distance between both surfaces. The figure 4 shows a representation of such a projection in image space.

Our model is consistent with the correlation between the CSF loss and the amount of deformation, studied by [4. The displacement norm of a point in the brain is an increasing function of the CSF volume lost. This property guarantees the convexity of the error as a function of the two CSF volume losses (see an example in Fig. 5p: a local minimum is a global minimum.

Even an efficient optimization method for our problem would require at least several tens of iterations to converge to an estimation of the parameters. Each iteration requires to compute a direct simulation, which takes time (more than 5 minutes) and therefore is not compatible with an application during a neurosurgical procedure. To speed up the intra-operative computation, we pre-compute before the surgery a set of brain configurations, deformed by a simulated brain shift for a regular sampling of the parameters domain. Then, during the procedure, a mesh is extracted from an intra-operative image, and compared to all the pre-computed deformed configurations. The configuration with the smallest error corresponds to the registered brain.

Finally, the displacement field enables to compute rapidly the displacement and deformation of other structures such as the ventricles, the target or the blood vessels.

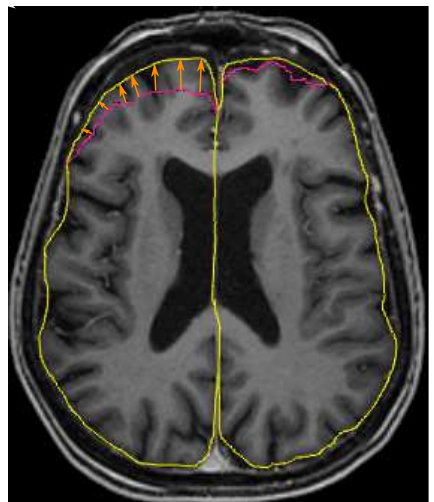

(a) Measure of similarity: the intra-operative triangular surface (pink) is projected on the simulated brain surface (yellow). The measure is the average distance of projection.

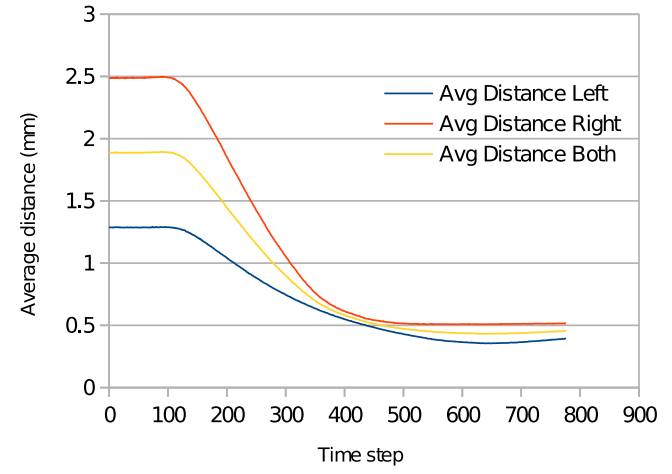

(b) At each time step of a simulation, the average distance between the simulated brain and the intra-operative data is measured. In this example, the simulation is parameterized with the same values used to generate the intra-operative data, that is why the distance converges to a small value.

Fig. 4: Mesure of similarity between the simulated brain state and intra-operative data. 


\section{Results}

To validate our method, we first apply it on a template containing high-fidelity models of the brain, skull, skin, ventricles and blood vessels. In our tests, both hemispheres are meshed with approximately 10,000 tetrahedrons. The Young's modulus $E$ is set to $3000 \mathrm{~Pa}$ and Poisson's ratio to 0.45 according to [6].

In this section, we show that our method is numerically sound. A virtual brain shift, obtained with fixed chosen parameters, is applied on the template. The obtained configuration will be used as a synthetic of 3D intra-operative data. These data are then used in the parameters estimation process to prove the consistency of the method: the estimated parameters are compared to the parameters used to generate the data.

Finally, we also show that if the Young's modulus differs slightly from the mechanical parameters used to generate the synthetic intra-operative mesh, our algorithm still register the intra-operative brain with a minimal error.

Data Generation With given input parameters $V_{L}$ and $V_{R}$ (CSF loss volume for both sides), a deformation is computed with the brain shift simulation: the pre-operative configuration undergoes a CSF leak leading to a brain shift. After the brain shift, when the deformed brain is at equilibrium, the surface in contact with air is extracted, i.e. the same data we extract from intra-operative patient data (see pink line in Fig. 1). The surface is uniformly remeshed with a marching cube algorithm to mimic data coming from 3D images, and noise is added, to meet as much as possible the intra-operative conditions. Finally, the parameters leading to this surface are estimated.

Validation protocol Multiple intra-operative data were generated in order to validate different scenarios: symmetric brain shift(\#1), asymmetric brain shift (\#2), unknown mechanical parameters for both symmetric (\#3) and asymmetric brain shifts (\#4). The parameters (CSF loss volumes and Young's modulus) of each scenario are summarized in the table 1.

\begin{tabular}{c|c|c|c|c|c|c|c|c}
\hline & \multicolumn{4}{|c|}{ Input parameters } & \multicolumn{4}{c}{ Optimization output } \\
\hline & Volumes $\left(\mathrm{cm}^{3}\right)$ & \multicolumn{1}{|c}{ Young's modulus(Pa) } & \multicolumn{2}{c}{ Volumes $\left(\mathrm{cm}^{3}\right)$} & \multicolumn{1}{|c}{ Average distance $(\mathrm{mm})$} \\
\hline Scenario & Left & Right & $E_{\text {data }}$ & $E_{\text {simu }}$ & Left & Right & Left & Right \\
\hline$\# 1$ & 30 & 30 & 3000 & 3000 & 30 & 30 & 0.4 & 0.4 \\
$\# 2$ & 10 & 20 & 3000 & 3000 & 10 & 20 & 0.5 & 0.5 \\
$\# 3$ & 30 & 30 & 3000 & 4000 & 40 & 40 & 0.4 & 0.4 \\
$\# 4$ & 10 & 20 & 3000 & 4000 & 15 & 25 & 0.6 & 0.5 \\
\hline
\end{tabular}

Table 1: Different scenarios of brain shift used to validate the method. The scenarios \#2 and \#4 mimic an asymmetric brain shift. \#3 and \#4 use data generated with mechanical parameters different from the minimization process. 

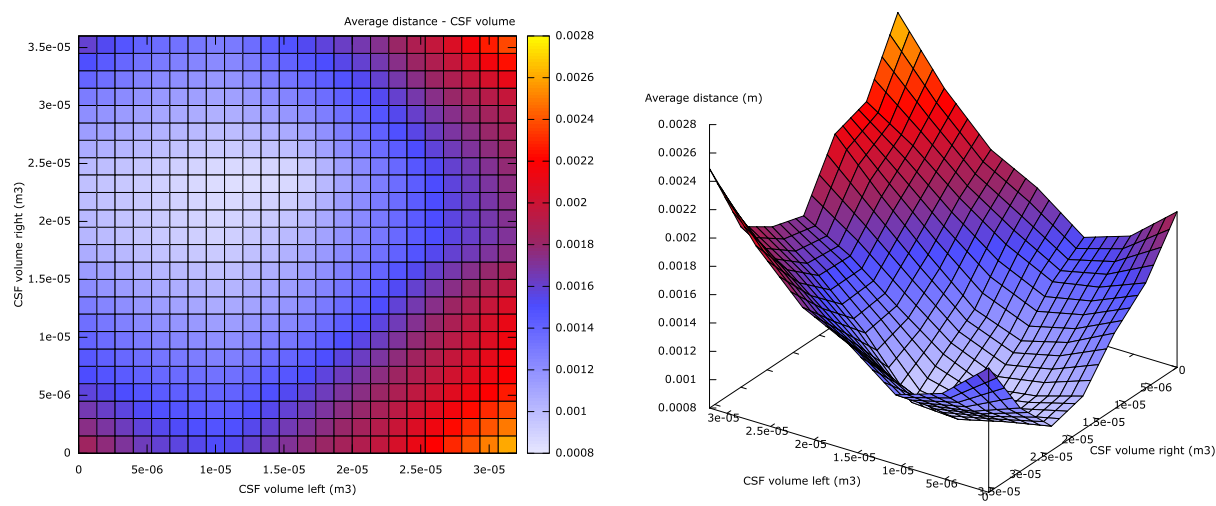

Fig. 5: The CSF loss volume are varying ( $\mathrm{X}$ and $\mathrm{Y}$ axis). The colors represents the average distance between the equilibrium state of the deformed brain caused by a CSF loss $(\mathrm{x}, \mathrm{y})$. The minimum is located at $\left(1 \times 10^{-5}, 2 \times 10^{-5}\right)$, which is the set of parameters used to generate the intra-operative data (scenario \#2).

Error measurement For each scenario, a set of deformation is computed, and compared to the synthetic intra-operative model, in order to find the minimum distance. The figure 5 shows the average distance between data generated with the scenario \#2 and the simulated brain shifts, with CSF losses varying between 0 and $30 \mathrm{~cm}^{3}$.

The table 1 summarizes the error between the values used to generate the synthetic intra-operative model, and the values estimated by the minimization process. These values are comparable only if the mechanical parameters used for generation and optimization process. To measure errors if the mechanical parameters are different, we compare directly the brain geometry with the measure of similarity presented in the section 2.2 .

The data in the table 1 show that, in the scenarios \#1 and \#2, the volume parameters are the same in input and output (the error in the measure of similarity comes from the remeshing and noise in the intra-operative data). In scenarios \#3 and \#4, where the brain is stiffer in the parameters estimation, the volumes estimated are greater, as expected, but the brain deformed with these parameters still matches the intra-operative data. We conclude that the Young's modulus does not have an effect on the registration output.

The figure 6 shows an example of the difference between the pre-operative configuration of the brain and the deformed brain fitting the intra-operative data. With our method, we are able to estimate the CSF volume lost, which gives a registration of the brain if used in the brain shift model. The entire process is based on a physical model, and no artificial forces are needed. The registration algorithm also provides the deformation and displacement of brain structures thanks to the displacement field. 

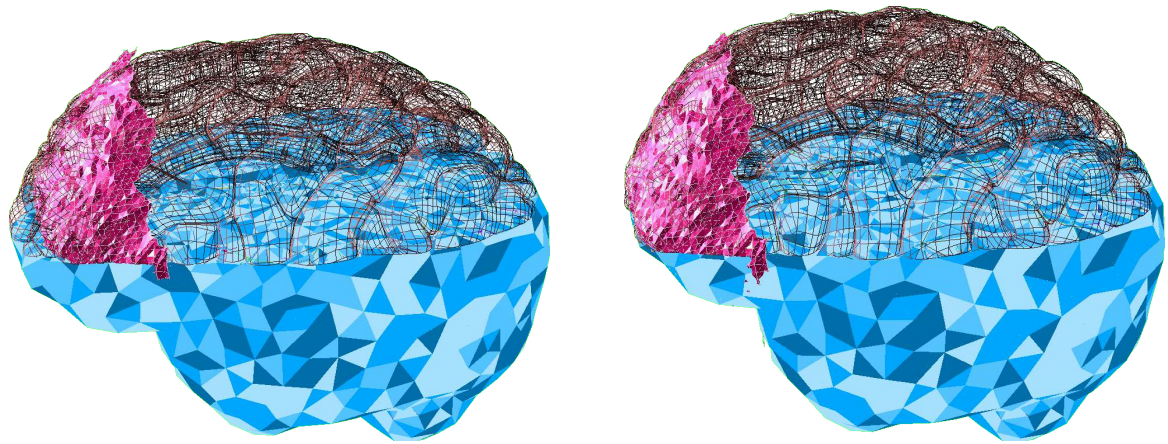

Fig. 6: 3D view of the mechanical model of the brain (in blue, only cut for visualization purpose). In pink, the mesh corresponding to intra-operative data. Left: the mechanical model is in its pre-operative configuration. Right: The brain fits the intra-operative data after a deformation

\section{Conclusion}

In this paper, we presented a method to register the pre-operative configuration of the brain onto the intra-operative state. The registration is entirely based on physics and handle patient-specific geometry, patient's head orientation and asymmetry in the deformation. We showed the numerical consistency of the method on 4 scenarios involving symmetry/asymmetry and mechanical parameters changes. The algorithm provides also the physical displacement and deformation of other structures such as the blood vessels or the target. The method is independent from the constitutive law of the brain, and could be improved using a more accurate law. The next step of this work is to validate it on a series of patient.

\section{Acknowledgment}

The authors would like to thank the French Research Agency (ANR) for funding this study through the ACouStiC project.

\section{References}

1. Audette, M.a., Siddiqi, K., Ferrie, F.P., Peters, T.M.: An integrated range-sensing, segmentation and registration framework for the characterization of intra-surgical brain deformations in image-guided surgery. Computer Vision and Image Understanding 89(2-3), 226-251 (Feb 2003)

2. Bilger, A., Duriez, C., Cotin, S.: Computation and visualization of risk assessment in deep brain stimulation planning. Studies in health technology and informatics 196, 29-35 (Jan 2014) 
3. Chen, I., Coffey, A.M., Ding, S., Dumpuri, P., Dawant, B.M., Thompson, R.C., Miga, M.I.: Intraoperative brain shift compensation: accounting for dural septa. IEEE transactions on bio-medical engineering 58(3), 499-508 (Mar 2011)

4. Elias, W.J., Fu, K.M., Frysinger, R.C.: Cortical and subcortical brain shift during stereotactic procedures. Journal of neurosurgery 107(5), 983-8 (Nov 2007)

5. Felippa, C., Haugen, B.: A unified formulation of small-strain corotational finite elements: I. Theory. Computer Methods in Applied Mechanics and Engineering 194(21-24), 2285-2335 (Jun 2005)

6. Ferrant, M., Nabavi, A., Macq, B., Black, P.M., Jolesz, F.a., Kikinis, R., Warfield, S.K.: Serial registration of intraoperative MR images of the brain. Medical image analysis 6(4), 337-59 (Dec 2002)

7. Sellier, M.: An iterative method for the inverse elasto-static problem. Journal of Fluids and Structures 27(8), 1461-1470 (Nov 2011)

8. Skrinjar, O., Nabavi, A., Duncan, J.: Model-driven brain shift compensation. Medical image analysis 6(4), 361-73 (Dec 2002)

9. Slotty, P.J., Kamp, M.a., Wille, C., Kinfe, T.M., Steiger, H.J., Vesper, J.: The impact of brain shift in deep brain stimulation surgery: observation and obviation. Acta neurochirurgica (Aug 2012)

10. Wittek, A., Miller, K., Kikinis, R., Warfield, S.K.: Patient-specific model of brain deformation: application to medical image registration. Journal of biomechanics 40(4), 919-29 (Jan 2007) 